\title{
An efficient method of sub- signal channel modulation on hitless redundancy switching systems
}

\author{
Takahiro Kubo $^{1 \text { a) }}$, Yuhei Kawakami ${ }^{1}$, \\ Go Yazawa ${ }^{1}$, Natsuki Yasuhara ${ }^{1}$, Shinichi Yoshihara ${ }^{1}$ and \\ Tomoaki Yoshida ${ }^{1}$ \\ ${ }^{1}$ NTT Access Network Service Systems Laboratories, NTT Corporation, \\ Midori-cho, Musashino-shi, Tokyo 180-8585, Japan \\ a) takahiro.kubo.mz@hco.ntt.co.jp
}

Abstract: In the hitless redundancy switching system, the selector of the redundancy switching device is controlled on the basis of the control information such as the connection status of the path to transmit frames, end-to-end delay of the path, and jitter. However, the control signal transmitted superimposed on the main signal channel in the hitless redundancy switching system is not highly confidential because all the information is transmitted superimposed on the two paths. In our previous study, we modulated the transmission timing of frames in the main signal channel by assigning " 0 " and " 1 " codes to the two paths in the hitless redundancy switching system. In this paper, we propose a method to efficiently modulate sub-signal channels in a hitless redundancy switching system to improve the communication bandwidth, and evaluate its effectiveness in a numerical simulation.

Keywords: hitless, redundant path network, Ethernet

Classification: Network system

\section{References}

[1] IEEE 802.1Q-2018, "IEEE Standard for Local and Metropolitan Area Networks - Bridges and Bridged Networks,” May 2018.

[2] Y. Sakagami, S. Taniguchi, and R. Kawate, "A Study and Evaluation on Hitless Switching Scheme for Dedicated Ethernet," in IEICE Technical Report, CS2013-1, pp. 1-5, Apr. 2013.

[3] G. Yazawa, T. Kubo, N. Yasuhara, and S. Yoshihara, "Efficient resource allocation method for hitless redundancy switching technology at multiple sites," IEICE Communication Express, vol. X10-B, no. 8, pp.-, Aug. 2021.

[4] Y. Luo, H. Roberts, K. Grobe, M. Valvo, D. Nesset, K. Asaka, H. Rohde, J. Smith, J. Wey, and F. Effenberger, "Physical Layer Aspects of NG-PON2 Standards_-Part 2: System Design and Technology Feasibility [Invited]," $J$. Opt. Commun. Netw., vol. 8, pp. 43-52, 2016.

[5] K. Honda, H. Nakamura, K. Hara, K. Sone, G. Nakagawa, Y. Hirose, T. 
Hoshida, and J. Terada, "Wavelength control method of upstream signals using AMCC in WDM-PON for 5G mobile fronthaul," Opt. Express, vol. 27, pp. 26749-26756, 2019.

[6] Y. Kawakami, T. Kubo, G. Yazawa, N. Yasuhara, and S. Yoshihara, “A Study of Communication System Using Transmission Timing to Redundant Path Network,” IEICE Gen. Conf. '20, B-8-45, March 2020.

\section{Introduction}

Wide-area Ethernet has become a useful option for low-cost communication between multiple locations. In wide-area Ethernet services, networks are used to increase availability. Hitless redundancy switching systems (HRSS) have been discussed that transfer frames to multiple paths for communication without interruption [1-3].

In HRSS, the selector of the redundant switching device is controlled on the basis of the control information such as the connection status of the path to transmit frames, end-to-end (e2e) delay of the path, and jitter. In general, the control signals to control the device itself are sent on a separate channel from the main signal channel where user data is sent [4-5], and the control signals to control the transfer of user data between devices are often sent superimposed on the main signal channel. The control signals exchanged between devices are also sent by superimposing them on the main signal channel as they are, so it is difficult to say that the system has excellent confidentiality. However, if the control signals can be deciphered only by combining the information of both control signals transmitted over two paths, high-security transmission can be achieved by taking advantage of the characteristics of the HRSS. However, to the best of our knowledge, there have been few reports on the communication of such control signals.

We previously proposed a method to transmit binary code information separately from data in user frames by modulating the transmission timing of frames in the main signal channel with a code of " 0 " and " 1 " for each of the two paths in a HRSS and having the receiver first arrive at the main signal channel frame of the intended path by a sequence number [6].

In this paper, we propose a method to efficiently modulate sub-signal channels in HRSS to improve the communication bandwidth and evaluate its effectiveness by numerical simulation.

\section{Sub-signal channel multiplex on HRSS}

\subsection{Conventional HRSS}

A conventional HRSS with a two-route redundant configuration consists of a pair of redundancy switching devices as shown in Fig. 1. Each device has one port that connects to the user NW and two ports that connect to the relay NW. Let us consider sending from user \#1 to user \#2 using the HRSS. Redundancy switching device \#1 assigns a sequence number to the frame received from user \#1, duplicates it, and sends it to NW \#1 and NW \#2. Redundancy switching device \#2 
checks the frames received from the respective relay NWs and confirms the order of arrival of frames with the same sequence number. The first arriving frame is sent to the user NW, and the second arriving frame is discarded. With the above operation, the HRSS provides uninterrupted communication between users \#1 and \#2 even if one of the routes becomes disconnected.

(a)

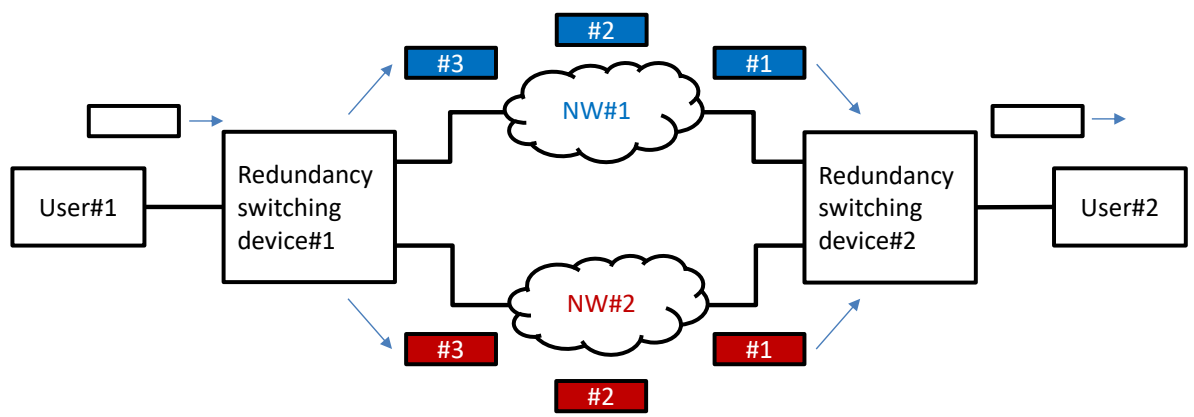

(b)
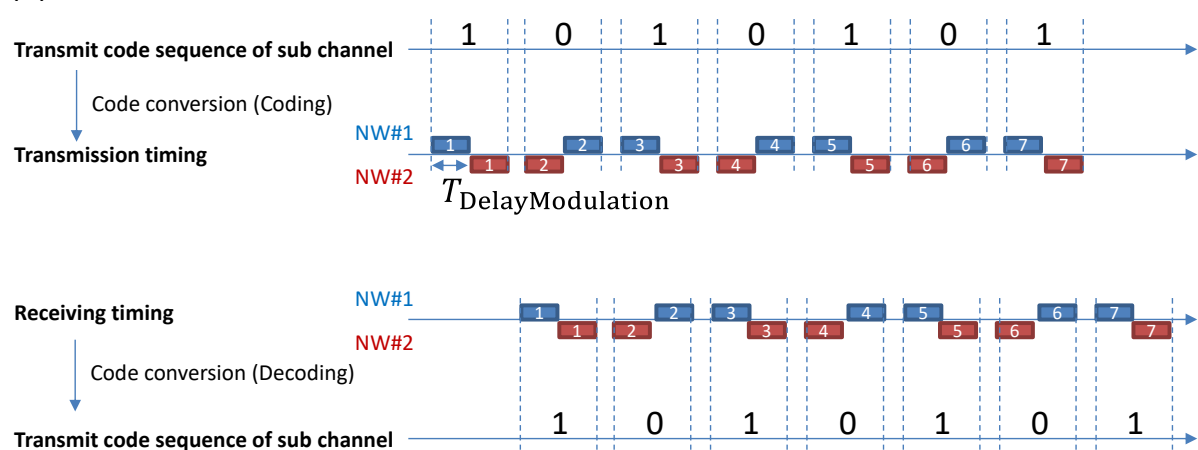

Fig. 1. Communications system using transmission timing on redundant path network.

\subsection{Proposed sub-signal channel modulation method}

The proposed sub-signal channel modulation method is shown in Fig. 1(b). The main signal channel and the sub-signal channel are superimposed at layer 1.

The main signal channel duplicates the user frame at redundancy switching device \#1 and forwards it to redundancy switching device \#2 via two relay NWs, which is the basic function of the HRSS. The sub-signal channel transmits control signals. Figure 1(b) shows, as an example, a case where a control signal with the code sequence "1010101" is transmitted.

The sub-signal channel does not prepare the bits to be transmitted as in the user frame of the main signal channel. Instead, each bit of the control signal is composed of two frames that are duplicated to transfer the redundant path in the main signaling channel, and the transmission timing is adjusted so that the intended arrival order is achieved upon reception. In Fig. 1(b), the code conversion is performed so that the transmission timing at which NW\#1 arrives first is "1," and the transmission timing at which NW\#2 arrives first is "0." To ensure that the signal of the intended path arrives first, redundancy switching device \#1 delays the frame transmission time of the path to be arrived at later by $T_{\text {DelayModulation. }}$. For example, the first code "1" of the sub-signal channel in Fig. 1(b) delays the frame 
transmission time of sequence number 1 of NW \#2 by $T_{\text {DelayModulation }}$ to control the frame of sequence number 1 of the main signal channel so that the frame via NW \#1 arrives first. Redundancy switching device \#2 decodes the information of the sub-signal channel by judging the port number (routes) to which the frame with the same sequence number arrived first as a binary code. We note that the main signal transmits continuously in order for the sub signal to transmit.

For transmission using the above method, the modulation speed $v$ of the sub-signal channel is expressed by the following equation

$$
v=\frac{\alpha}{\left(\beta+\alpha \times T_{\text {DelayModulation }}\right)}
$$

where $\alpha$ is the wire rate of the main signal channel, $\beta$ is the bit length of the user frame, and $T_{\text {DelayModulation }}$ is the delay time interval. From eqn. (1), we can see that the modulation rate of the sub-signal channel can be increased by keeping $T_{\text {DelayModulation }}$ small.

$T_{\text {DelayModulation }}$ can be set to a sufficiently small value when the difference between the average path delays of NW \#1 and NW \#2, $\mid T_{\text {DelayNW\#1 }}-$ $T_{\text {DelayNW \#2 }} \mid$, is zero and the jitter can be regarded as zero, because the intended reception timing will not be shifted.

On the other hand, if $T_{\text {DelayModulation }}$ is $\left|T_{\text {DelayNW\#1 }}-T_{\text {DelayNW \#2 }}\right|>0$, then $T_{\text {DelayModulation }}>\left|T_{\text {DelayNW\#1 }}-T_{\text {DelayNW \#2 }}\right|$ must be satisfied to prevent the intended reception timing from shifting due to the difference in propagation delay caused by the different routes.

Figure 2 shows the timing when redundancy switching device \#1 sends frames to each path and the timing when redundancy switching device \#2 receives frames from each path when NW \#1 has a larger average path delay than NW \#2. Here, the code sequence transmitted by the sub-signal channel is assumed to be the "1010101" signal.

Figure 2(a) shows an existing timing adjustment method in which frames to be post-delivered are transmitted with a $T_{\text {DelayModulation }}$ delay. The reception timing is the intended reception timing in each code. However, improvement is needed considering that the difference between the two path lengths for forwarding the frames is large enough to reduce $v$.

Figure 2(b) shows the proposed reception timing correction method. In this method, the reception timing of a frame received from a path with a short average path delay difference is delayed by the average path delay difference from a path with a long average path delay difference. As a result, since the average path delay difference can be excluded from the value of $T_{\text {DelayModulation, which delays the }}$ frame to be post-delivered, the modulation speed $v$ of the sub-signal channel can be increased by setting $T_{\text {DelayModulation }}$ to a smaller value than in Figure 2(a). 
(a)

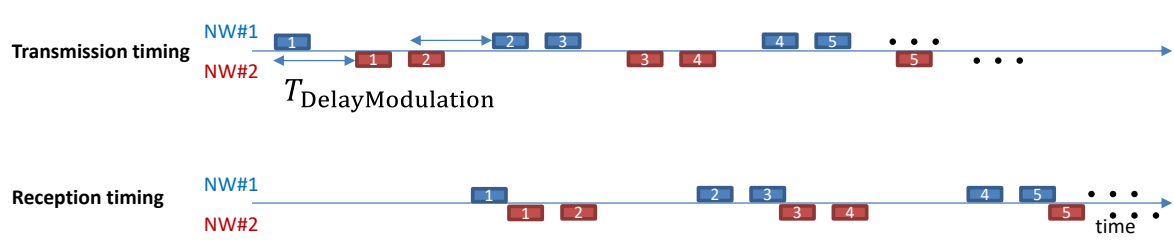

(b)

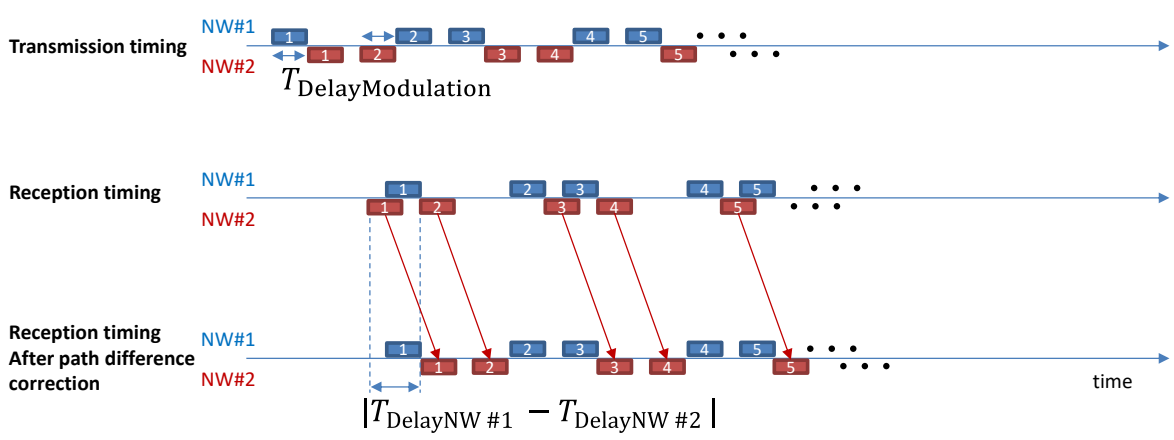

Fig. 2. Proposed reception timing correction method.

\section{Numerical analysis}

To evaluate the effectiveness of the proposed method, we calculate the existing modulation rate $v$ and the proposed modulation rate $v^{\prime}$ of the sub-signal channel in a HRSS. Since the average path delay difference is $100 \mathrm{msec}$, the delay time interval $T_{\text {DelayModulation }}$ of $v$ is $100 \mathrm{msec}$. On the other hand, $v^{\prime}$ does not need to take the average path delay difference into account. Then, $T_{\text {DelayModulation }}$ of $v^{\prime}$ is $1 \mathrm{msec}$ taking only the jitter into account. The code sequence of the sub-signal channel used for the evaluation is a code sequence of alternating " 1 " and " 0 " such as "1010...10". Figure 3(a) shows the results of evaluating the values of transmission speeds $v$ and $v^{\prime}$ of the sub-signal channel when the value of $\beta$ is changed from 64 to 1522 bytes when the wire rate of the main signal channel is $\alpha=1 \mathrm{Gbps}$ with values of $T_{\text {DelayModulation. }} v$ is about $10 \mathrm{~b} / \mathrm{s}$ because the value of the average path delay difference $(100 \mathrm{msec})$ is included in $T_{\text {DelayModulation. }}$ On the other hand, $v^{\prime}$ is about $1 \mathrm{~kb} / \mathrm{s}$ in the frame length range when considering only the jitter $(1 \mathrm{msec})$ because the value of the average path delay difference is not

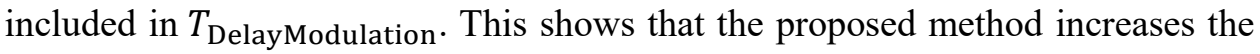
modulation speed by about 100 times compared with the existing method. 


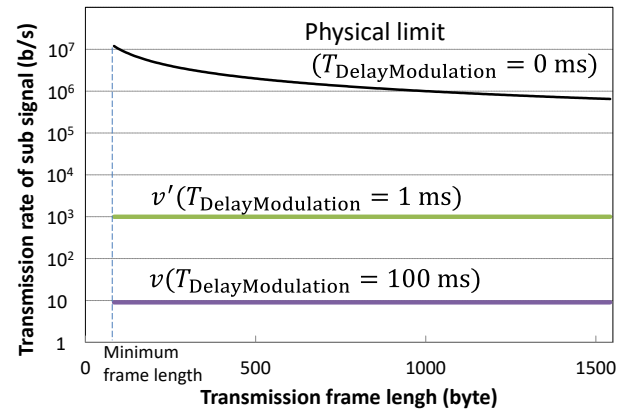

(a)

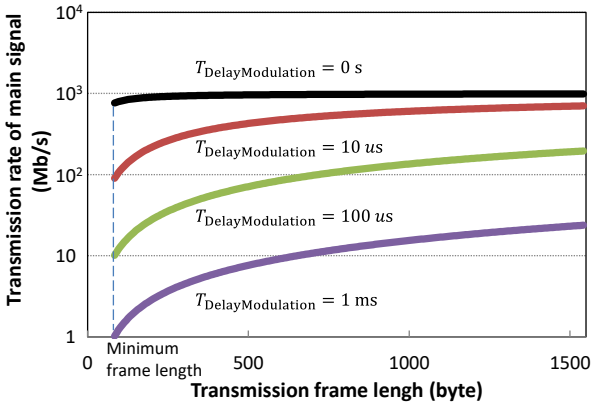

(b)

Fig. 3. Numerical results of transmission rate of (a) sub-signal channel and (b) main-signal channel.

The physical limit of the proposed method can be specified as the speed at $T_{\text {DelayModulation }}=0$, which is shown by the black line. The two factors that increase $T_{\text {DelayModulation }}$ are the average path delay difference and jitter. Then, we can see that the magnitude of jitter is the factor that determines the speed of the proposed method. In this evaluation, the jitter is assumed to be $1 \mathrm{msec}$, but the speed can be improved by using a path with even smaller jitter.

Next, to evaluate the impact of the proposed method on the main signal, we calculated the transmission rate of the main signal with the code sequence where the same code sequence occurs with probability $1 / 2$. Figure $3(\mathrm{~b})$ shows the transmission rate of the main signal with values of $T_{\text {DelayModulation. As shown in }}$ Fig. 3(b), the parameters that affect the speed of the main signal are $T_{\text {DelayModulation }}$ and frame length. In other words, in a system where the frame length of the main signal is long enough and the jitter is kept small, the impact of the proposed method on the main signal is minimal.

Finally, we discuss the bit error of the proposed method. In the proposed method, the bit error is caused by the reversal of the arrival order of frames with the same sequence number received from each path, so that one frame order reversal is a 1-bit error. The bit rate of the sub-signal channel is equal to the frequency of frames that receive a delay greater than the value of $T_{\text {DelayModulation }}$. To suppress bit errors, we can take two approaches. One is to use the proposed method in a delay-guaranteed network where the maximum delay is guaranteed. Another is to optimize value of $T_{\text {Delay Modulation }}$ according to jitter of networks.

\section{Conclusion}

We proposed a method to efficiently modulate the sub-signal channel in a HRSS to improve the modulation rate by reflecting the processing related to the average path delay difference in the timing. Numerical simulations show that the proposed method significantly increases the modulation rate compared with the existing methods. 